\title{
Civilisations
}

Revue internationale d'anthropologie et de sciences

humaines

$54 \mid 2006$

Expériences de recherche en République

démocratique du Congo

\section{Etre et avoir à Kinshasa}

Expériences de reportage informel au Congo-Zaïre

Jean-François Bastin

\section{(2) OpenEdition}

Journals

Édition électronique

URL : http://journals.openedition.org/civilisations/454

DOI : $10.4000 /$ civilisations. 454

ISSN : 2032-0442

Éditeur

Institut de sociologie de l'Université Libre de Bruxelles

Édition imprimée

Date de publication : 1 avril 2006

Pagination : 199-208

ISBN : 2-87263-006-6

ISSN : 0009-8140

Référence électronique

Jean-François Bastin, « Etre et avoir à Kinshasa », Civilisations [En ligne], 54 | 2006, mis en ligne le 01

avril 2009, consulté le 07 mai 2019. URL : http://journals.openedition.org/civilisations/454 ; DOI :

$10.4000 /$ civilisations.454

(c) Tous droits réservés 


\title{
Etre et avoir à Kinshasa \\ Expériences de reportage informel au Congo-Zaïre
}

\author{
Jean-François BASTIN
}

Résumé : Expériences de reportage pour la radio-télévision belge au Congo de 1980 à 2003. Il n'y a pas de progression logique dans le déroulement des faits mais une succession d'expériences originales, discontinues, peu prévisibles, témoignant sans doute d'un fonctionnement similaire de la société congolaise, souvent qualifié d'informel. Cette succession d'expériences pose une question lancinante : comment voir sans être vu? Comment observer sans être soi-même observé, sans déranger l'ordre et le désordre des choses? Est-il possible pour des Belges de filmer des Congolais, sans qu'interfère leur passé commun? La vraie constante dans ces expériences est la relation des Congolais à la caméra professionnelle. Celle-ci est surtout perçue comme un objet étranger, blanc, extérieur, comme une caméra de surveillance, un objet décidément post-colonial, face auquel il faut se composer une attitude de défi et de fierté. L'auteur propose d'organiser ses tournages comme un échange, un donnant-donnant, où l'argent occupe une place importante, mais pas essentielle : qu'ils soient payés ou pas, les Congolais filmés sont finalement les seuls à décider de ce qu'ils donnent à la caméra. En contrôlant leur image, ils s'approprient une part du film.

Mots-clés : filmer, reportage, représentation, échange, insoumission.

Summary: Experience as reporter for Belgian radio and television in Congo between 1980 and 2003. There is no logical progression in the unfolding of events but a succession of original, fragmented, unpredictable experiences probably testifying to a similar functioning of Congolese society, usually qualified as informal. This succession of experiences raises some troublesome questions: How to see without being seen? How to observe without yourself being observed, without upsetting the order and disorder of things? Is it possible for Belgians to film Congolese without their common past getting in the way? The unwavering fact in these experiences is the relationship that Congolese have with a professional camera. This thing is perceived as a foreign, white, outside object, like a surveillance camera, a resolutely post-colonial object, opposite which an attitude of defiance and pride is necessary. The author organises his reports like an exchange of give-and-take where money plays an important - but not essential - role. Whether they are paid or not, filmed Congolese are ultimately alone in deciding what they give to the camera. By controlling their image, they appropriate part of the film.

Key words: filming, reporting, representation, exchange, resistance. 
Cette contribution sera une sorte de compte-rendu, un récit chronologique, plus par commodité que par nécessité. Car il n'y a pas de progression logique dans le déroulement des faits. Il y a une succession d'expériences originales, discontinues, peu prévisibles, témoignant sans doute d'un fonctionnement similaire de la société congolaise. Le récit sera entrecoupé de quelques réflexions plus générales sur cette sociologie de l'apparition, sur la manière de se présenter à l'œil européen (belge très précisément) et sur les rapports particuliers qui s'instaurent entre le filmeur belge et le filmé congolais.

\section{Juin 1980}

$\mathrm{Je}^{1}$ suis à Kinshasa avec mon magnétophone. Je prépare pour le journal parlé de la Radio et Télévision Belge Francophone (RTBF) une série d'émissions sur l'indépendance du Congo, devenu Zaïre. Je suis curieux de retrouver cette ville que j'ai quittée il y a vingt ans. Je suis impatient de rencontrer ceux qui ont « fait » cette indépendance, restée dans ma mémoire d'enfant comme une aventure exaltante et mystérieuse. Mon but est très simple : enregistrer les témoignages des principaux acteurs sur les événements majeurs de la décolonisation. Ce reportage est un fiasco. Toutes les portes se ferment. Les témoins se dérobent. Personne ne veut parler $^{2}$. A ce grand silence, une seule explication. Nous sommes en 1980, l'année du retour de la répression, de la dictature féroce après trois années de flottement du mobutisme qui ont permis la naissance d'une vague opposition démocratique. Aucun ordre n'est venu du Palais de marbre pour faire taire tous ces gens, mais la peur est partout. Personne ne veut prendre le risque de se faire valoir, d'égratigner la légende du Guide libérateur de la nation. L'autocensure est préventive et absolue.

\section{Juin 1985}

L'indépendance est encore le prétexte de ce reportage, mais tout est différent. Je débarque à Kinshasa avec une équipe de télévision ${ }^{3}$, douze jours avant le 30 juin, dix jours avant l'arrivée du Roi et de la Reine des Belges. Mobutu joue son va-tout avec ce $25^{\mathrm{e}}$ anniversaire : il amnistie les leaders de 1'Union pour la Démocratie et le Progrès Social (UDPS) relégués depuis des années, il multiplie les gages à l'égard de l'Occident et de la Belgique. Le Roi se pince le nez et accepte de revenir au « Congo », quinze ans après son idyllique voyage de 1970. Mobutu va s'emparer de ce cadeau royal. Nous sommes résolus à profiter des circonstances, de ce besoin impérieux de reconnaissance par la Belgique. Nous misons sur l'ambiance des retrouvailles ${ }^{4}$, sur un relâchement

1. J'utiliserai le « je » par commodité. Parce que « je » suis le seul élément de continuité des expériences qui vont être évoquées. Pourtant, à part pour la première, radiophonique, je n'ai jamais été seul à les vivre. En télévision, un reporter ou un documentariste n'est jamais seul, le travail est toujours collectif. Le « je » se fera « on » ou « nous », sans importance et sans règle précise.

2. Al'exception du vieil écrivain Lomami Tshibamba qui raconte la chicote, le racisme tranquille et les failles notamment religieuses - du système colonial, et de l'ex journaliste, ex-Prix Pulitzer, Gabriel Makosso qui raconte les événements de 1956 à 1960.

3. Voir filmographie en annexe.

4. Le général Janssens lui-même, le désastreux commandant en chef de la Force publique en 1960, est de retour, invité d'honneur de Mobutu. 
de la répression, sur une certaine modération des coureurs de matabiche, ce bakchich congolais. Nous faisons du reportage de terrain, aux quatre coins de la capitale, mais nous biaisons : nous ne travaillons pas sur l'actualité, sur la situation politique, sur la nature du régime, nous travaillons sur la ville, sur la misère, la créativité, la fureur de vivre kinoise. Vingt-cinq ans après l'indépendance, alors que se multiplient les bilans désastreux de la décolonisation, l'idée est de faire (re)découvrir Kinshasa et ses habitants au public belge, notamment à ceux qui ont connu Léopoldville à l'époque de sa «splendeur» coloniale, de regarder autrement ${ }^{5}$ ces gens et cette ville qui ont tant compté dans l'histoire de Belgique.

Mais comment filmer les Kinois sans les interférences constantes de ce passé, de cette histoire? Comment voir sans être vu? Comment observer sans être soi-même observé, scruté, interpellé, sans déranger l'ordre et le désordre des choses? Comment filmer sans être partie prenante de ce qui est filmé? Au fond, est-il possible pour des Belges de filmer des Congolais? Des Blancs peuvent-ils innocemment filmer des Noirs, comme si de rien n'était? Je me rappelle que dans les reportages de la RTBF au Zaïre dans les années 1970 (pour les émissions 9000009 et $A$ Suivre), le tutoiement était souvent d'usage à l'égard des Congolais. Justifié par une sorte d'évidence naturelle («Au Zaïre, tout le monde se tutoie »), ce tutoiement n'était évidemment pas entendu en Belgique comme au Zaïre. Les reporters faisaient comme s'ils n'étaient pas Belges travaillant pour un public belge et comme si le tutoiement n'avait pas une histoire coloniale (d'ailleurs évoquée par Lumumba dans son discours du 30 juin 1960).

Pour affronter ces questions, toujours valables vingt ans plus tard, je choisis d'en faire un des sujets et finalement le sujet principal du film. Le reportage est un jeu de miroirs. Les Kinois posent ouvertement, ils se présentent à la caméra, ils nous interpellent : «Vous filmez? Pourquoi vous filmez? Nous distribuons des ballons, des autocollants, des tee-shirts siglés RTBF et nous les filmons à l'occasion, sur les gens, dans la ville, comme la signature flagrante de notre interférence. Nos personnages sont des figures de la vie kinoise devenues depuis assez classiques (catcheur, sentinelle, handicapé, musicien, coiffeuse, jeune fabricant d'objets en fil de fer). On nous sent très proches, mais je les vouvoie bien sûr... Nous sommes parfois dans l'image. Le caméraman a pour consigne de filmer ce qui se passe « réellement », c'est-à-dire notamment la confrontation filmeursfilmés. La séparation entre les uns et les autres est brouillée. Nous sommes très loin de la fiction habituelle du reportage qui voudrait qu'on oublie la caméra ${ }^{6}$.

Le personnage clef est Makenga, notre chauffeur-accompagnateur-interprète, sorte d'agent double puisqu'il est des deux côtés de la barrière. Je l'interroge à plusieurs reprises, il est le commentateur, le chroniqueur du tournage : il explique, critique, ajoute son grain de sel. Dans le film, je l'appelle l' « observateur privilégié ». Il est rare qu'un film (reportage ou documentaire) dévoile à ce point son " procès de production » comme diraient les marxistes. Mais il y a tout de même un fameux non-dit : l'argent. D'emblée, j'ai décidé de rétribuer les « acteurs » de ce reportage. Je me situe dans un rapport d'échange,

5. La revue Autrement publie précisément, en cette même année 1985, un numéro sur les «capitales de couleurs » dont Kinshasa, qui inspirera en partie ce tournage.

6. «Faites comme si on n'était pas là » est une phrase courante dans la bouche d'un caméraman, entretenant l'illusion d'un réel pur, vierge de toute mise en scène, espérant sans doute que cette consigne s'appliquera au téléspectateur au moment de la diffusion. 
je considère que ce qu'ils offrent à la caméra a un prix. Je paie généralement en francs belges. Des sommes modiques mais suffisantes pour qu'il n'y ait pas de marchandage. L'initiative de cet échange vient toujours de moi. Cet argent est un signe de reconnaissance, sous la seule forme qui me semble valable dans cette relation ambiguë, lourde de toute l'histoire belgo-congolaise (euro-africaine). Peut-être est-ce uniquement dans ma tête que cela se passe, mais j'ai l'impression profonde qu'il sera longtemps difficile, voire impossible, d'avoir entre ex-colonisateurs et ex-colonisés des rapports totalement dénués de peur, de méfiance, de culpabilité et d'attentes inavouées. Et ceci est sans doute la raison principale de ce non-dit, de cette dissimulation particulière. Il y a probablement aussi la crainte d'être mal compris en Europe, d'être accusé de malversations journalistiques et documentaires. Il m'a toujours semblé que le débat suscité en France et dans le milieu documentaire européen autour du film « Etre et avoir $»^{7}$, par les exigences financières, a posteriori, de son héros principal, instituteur républicain de son état, serait inconcevable dans la plupart des pays d'Afrique : comment imaginer qu'une telle vedette puisse ne pas se faire payer et qu'un tel héros puisse ne pas être rémunéré alors que le film a rapporté gros?

Cette rétribution des «acteurs" sera désormais une ligne de conduite pour les reportages et documentaires ultérieurs, à l'exception évidente et notable de ceux qui vont suivre et seront de nature immédiatement politique.

\section{Octobre 1991 - Novembre 1994}

C'est le commencement de la fin pour le mobutisme. L'actualité politique commande des reportages politiques d'actualité. "Le léopard blessé », en 1991, est une sorte d'interview-poursuite du Président Mobutu retranché dans sa résidence de Nsele et sur son bateau Kamanyola. La faisabilité de ce genre de tournage dépend forcément du bon vouloir présidentiel mais également de son entourage, dont les contours sont flous et vastes. L'entourage peut retarder les choses, rarement les empêcher. On est ici dans un travail journalistique assez normal, sans spécificité particulière, qui serait réductible à une dimension africaine, congolaise ou kinoise. Pas question d'argent, à peine de pistons : les contacts utiles la veille peuvent être périmés le lendemain. On arrive ou on n'arrive pas jusqu'à Nsele selon des circonstances aléatoires. Une fois sur place, en présence du Chef, il n'y a plus d'entourage qui compte, il n'y a plus que le face-à-face de l'interview, et la caméra qui profite de la situation pour ingurgiter toutes les images qui lui sont généralement interdites.

En 1994, pour « Maréchal, revoilà ! », on est dans le même type de configuration. Il s'agit à nouveau d'approcher au plus près Mobutu, retranché cette fois à Gbadolite, pour observer son fonctionnement et sa capacité de peser encore sur les événements, intérieurs et extérieurs. Seule l'échelle est différente : en 1991, tout s'était passé en quelques heures; en 1994, le tournage s'étale sur deux bonnes semaines. Mobutu se multiplie, son entourage ou ce qu'il en reste vibrionne comme jamais : le Maréchal prépare son retour sur la scène internationale après trois ans d'opprobre, on va le suivre jusqu'à Nice puis à Biarritz où il est réinvité au sommet franco-africain. Le come-back fera pschitt, ce sera le dernier

7. Pour rappel, les exigences financières, a posteriori, de l'instituteur Lopez, héros central de ce documentaire à grand succès. 
voyage de Mobutu en Europe, le chant du cygne du Léopard. Mais à part cette différence d'échelle, les circonstances de tournage sont les mêmes. Une fois passés tous les barrages et contournés les entourages, on est dans l'échange classique entre reporters et leaders politiques : besoin de savoir, d'observer, de dévoiler d'un côté; besoin de se montrer et de communiquer de l'autre. Les uns et les autres pensant évidemment être les gagnants de cet échange ${ }^{8}$. Le reporter en l'occurrence se disant qu'il aura le dernier mot au montage.

Au fur et à mesure de ce tournage de 1994, la caméra devient de plus en plus distante, par la force des choses : une fois arrivé en France, Mobutu est accaparé par sa famille élargie puis par la presse internationale qui en fait la vedette médiatique du sommet franco-africain. Au final, le film de 60 minutes témoignera de cette énorme contradiction : Mobutu assailli par les médias et fui par ses pairs. Mobutu charismatique comme jamais, exilé tout puissant dans son propre pays, au comble de la célébrité internationale au moment de plonger dans la disgrâce définitive.

\section{De Mobutu à Kabila}

Le changement de régime, le passage du Zaïre à la RDC modifient les conditions de travail des journalistes et réalisateurs étrangers. Sous Mobutu, on fonctionnait dans un système relativement compréhensible. La corruption elle-même, généralisée, semblait répondre à des règles certes informelles mais assez constantes. Il y avait une hiérarchie de la corruption et personne ne perdait jamais de vue que l'on était en dictature. Pour pouvoir filmer à Kinshasa ou à Gbadolite, il fallait bien sûr se barder d'autorisations payantes, il fallait avoir un programme de tournage officiel, mais il était possible de travailler à peu près normalement, moyennant quelques solides palabres avec des autorités subalternes assez faciles à identifier (policiers et militaires surtout) ${ }^{9}$. Les laissez-passer, les signatures et cachets ministériels, en somme la peur des foudres mobutériennes avaient rapidement raison des pulsions lucratives des interpellateurs. D'autre part, il y avait une sorte de connivence de la population avec les reporters étrangers, qui permettait un certain travail clandestin, dont personne n'était vraiment dupe. Les interventions des fonctionnaires zélés tournaient souvent à l'engueulade générale propice à un repli en bon ordre, avec mise à l'abri des cassettes compromettantes. Tout cela n'allait pas toujours sans dégâts collatéraux. Ainsi, en 1985, après un tournage en ville avec le mendiant handicapé Félix que des policiers avaient essayé d'empêcher, ceux-ci se sont vengés en allant arrêter Félix, en le rançonnant et le coffrant pour la nuit ${ }^{10}$.

En deux mots, sous Mobutu, avec des hauts et des bas épousant les courbes de fluctuation des relations entre le Zaïre et la Belgique, il y avait des repères, on savait à peu près à quoi s'en tenir, de qui se méfier et comment s'en débarrasser. Sous Kabila,

8. En 1992, entre ces deux tournages centrés sur Mobutu, un reportage en Angola sur les « Tigres », les ex gendarmes katangais, participe d'une expérience parallèle, quoique relevant d'un autre type d'échange. Ici, au départ, l'intérêt des filmeurs et des filmés est pratiquement le même, leur intention est identique. Il y a la même envie de « scoop ». Nous voulons être les premiers à les filmer dans leur retraite, ils veulent se faire (re)connaître, ils veulent réexister dans l'histoire. Et le fait est qu'ils joueront bientôt un rôle militaire dans la reconquête du pays et la chute du mobutisme.

9. A l'exception très rare de civils se présentant, sans preuves, comme des cadres du parti unique.

10. Signalons aux âmes sensibles qu'en guise de dédommagement, nous avons offert à Félix un tricycle à manivelles qui lui a permis d'étendre son aire de travail. 
tous les repères sont brouillés. On passe de l'informel relatif à l'informel absolu, à l'imprévisibilité quasi totale. Il n'est plus possible de s'aventurer dans la ville sans une soigneuse préparation. Et encore : celle-ci peut toujours être prise en défaut.

Au début, beaucoup de journalistes trouvent que tout va mieux. Les reporters affluent. Les nouveaux dirigeants ont, par définition, l'attrait du neuf et bénéficient d'un préjugé favorable. Le problème, dans ce Congo new look, est que la société s'est brutalement émancipée. La peur ne vient plus d'en haut, elle vient de partout. Peur du vide, détresse, honte de soi, fuite des réalités (dans la religion notamment), hystéries multiples, fureur de vivre toujours plus rageuse, corruption dure, agressive, paranoïa xénophobe. Sortir une caméra dans cette anarchie générale est une gageure et une provocation. On ne peut plus se fier à un semblant d'ordre, fût-il totalement immoral. On ne peut plus compter que sur ses propres forces, on entre dans un jeu complexe de rapports de force multiples et impalpables.

En fait tout dépend de ce que l'on veut faire, du sujet du reportage ou du documentaire et, dès lors, des lieux de tournage. Le problème est tout différent si l'on tourne en intérieur ou en extérieur. Dans les deux cas, il faut une autorisation de tournage, mais dans le second cas cette autorisation, toujours nécessaire, ne sera jamais suffisante.

\section{Lubumbashi 1998}

Tournage simple, linéaire, intime pour «Missionnaire chez les Blancs », consacré à l'apostolat de nombreux prêtres congolais en Belgique. Nous raccompagnons dans sa famille lushoise Olivier Nkulu, vicaire à La Hulpe. A Lubumbashi, derrière les murs des maisons, des missions et des hôpitaux, on fait comme on veut. En ville, dans la rue, c'est une toute autre affaire. La caméra est immédiatement perçue comme un instrument d'espionnage. La RDC est en guerre, le Katanga est coupé de Kinshasa. Lubumbashi, comme le reste du Congo, vit dans la peur et la paranoïa. La ville est truffée d'hommes en armes, de kadogo, de militants portant brassard. L'Alliance des forces démocratiques pour la libération du Congo-Zaïre (AFDL) a remplacé, en plus voyant et en plus vigilant, le Mouvement populaire de la Révolution (MPR).

Les prises de vue extérieures sont assez rares, centrées pour la plupart sur notre personnage. Celui-ci joue simultanément le rôle d'acteur principal et de médiateur auprès de tous les interpellateurs, demandeurs d'autorisations et d'explications. Nous limitons au maximum ces tournages, peu importants au regard du sujet essentiellement réalisé en Belgique (il restera très peu d'images tournées à Lubumbashi dans le montage final). En fin de séjour, quand pratiquement tout est « en boîte », nous décidons de filmer quelques plans de ville pour répondre à d'éventuels besoins de montage. Parmi ces plans et par hasard, le siège rutilant, repeint à neuf, de l'AFDL. Il n'en faut pas plus pour passer la moitié de la nuit dans les locaux de la Sécurité, à essayer d'expliquer que cette image ne nous intéresse pas, qu'on peut l'effacer illico sur la bande. Il faudra une intervention du Consul de Belgique, venu rouvrir le poste quelques jours auparavant, pour nous sortir de cette impossible palabre. Notons qu'en ces débuts de révolution kabilienne, aucun de nos interrogateurs n'a fait mine de parler d'argent.

\section{Kinshasa 2000}

Je débarque à Kinshasa fin 1999 en compagnie de la réalisatrice Isabelle Christiaens et du journaliste radio de la RTBF François Ryckmans. Pour la première fois, j'ai décidé de faire un repérage, au plein sens du terme. Il s'agit d'abord de s'assurer qu'il sera 
possible de filmer en extérieur à Kinshasa quelques semaines plus tard. Le projet de film porte (comme il y a vingt ans en radio) sur l'indépendance. Nous voulons réaliser un documentaire pour le 30 juin 2000 qui s'intitulera «Indépendance cha cha », qui remémorera la vie quotidienne des Congolais dans les années cinquante et la création de la chanson mythique par Joseph Kabasele et l'African Jazz. Comparée à Kinshasa, Lubumbashi était un havre de quiétude et d'indifférence. Nous sommes rapidement convaincus qu'il faudra prendre des mesures importantes pour réussir ce tournage.

Le repérage nous permet d'identifier une série de personnages, de témoins qui nous guideront dans ce travail de mémoire (anciens combattants, historien, musiciens, anciens boys, ancien chef de quartier, etc.), mais aussi de contacter les nouvelles autorités pour les mettre au courant du projet et nous assurer de leur coopération. Outre de nombreux coins de Kinshasa, nous voulons en effet tourner dans des lieux normalement inaccessibles, pour raisons protocolaires et stratégiques (Mont Ngaliema, Palais de la Nation devenu la résidence de travail du Président Kabila). Le projet semble convenir à l'entourage présidentiel. Je parviens à établir un contact privilégié avec le Colonel Edy Kapend, l'aide de camp de Kabila, qui a la haute main sur les services de sécurité. Le repérage nous permet surtout d'engager pour la suite un collègue de la Radio et télévision nationale du Congo (RTNC), Paul Maseke, qui sait comment se fait un film et qui connaît Kinshasa comme sa poche.

Cette dernière initiative s'avérera essentielle lors du tournage, en janvier 2000. Avec Maseke, nous préparons systématiquement le terrain avant de sortir le matériel. Qu'il s'agisse du cimetière où est enterré Kabasele, de l'entrepôt de Kingabwa où sont entassées les vieilles statues coloniales, du rond-point Victoire ou de Lingwala, l'ex-quartier SaintJean, où nous voulons filmer les traces de l'apartheid colonial, partout nous prenons contact avec toutes les personnes susceptibles d'entraver le tournage et donc, si nous sommes assez prévenants, de le faciliter. C'est ainsi que nous pourrons filmer sans trop de problèmes, avec une sorte d'encadrement informel, circonscrit à des zones dont nous avons toujours du mal à mesurer l'étendue exacte. Les incidents restent limités. Ce travail de « déminage » suppose évidemment un budget matabiches, toujours plus facile à gérer préventivement et courtoisement qu'a posteriori, quand un incident a créé la pagaille et multiplié les envies de lucre.

Je me rappelle, lors d'un tournage en camion avec la fanfare " Confiance », que nous avions omis de «déminer » une petite portion du parcours. Cela nous a valu, à Maseke et moi-même une bonne heure de palabres au poste de police, pendant que l'équipe et la fanfare restaient immobilisées sur le camion. Nous n'avons rien décaissé, nous avons finalement bénéficié d'une intervention haut placée, un recours dont nous usions parfois, avec parcimonie : un homme des Services de renseignements, proche de Kapend, qui nous surveillait et veillait sur nous par la même occasion.

Je me rappelle encore que la journée de tournage à Lingwala, avec différents personnages, s'est terminée par une grosse bagarre au sein de la famille de l'ancien chef de quartier au temps colonial. Famille nombreuse et extensible comme il se doit, où se sont manifestés des gens frustrés et menaçants, se considérant lésés par nos arrangements.

Pour en revenir aux circonstances générales de tournage, notons que dans le cas de cet ancien agent colonial, comme de nos autres personnages, il n'est pas question de matabiches, mais de paiement de prestations. Les matabiches servent à faciliter le travail. Pour les personnages du film, c'est bien de rémunération qu'il s'agit, selon le principe adopté dès 1985, c'est-à-dire sans négociation préalable. La question financière n'est 
jamais abordée lors du premier contact. En ce qui concerne les prestataires de services, orchestre « Afric'Ambiance » ou fanfare « Confiance - Ecole belge », on peut parler d'un véritable engagement avec contrat négocié. Notons enfin, par souci de clarté, que toutes les séquences des films ne sont pas systématiquement "payantes ». Nous filmons par exemple au lycée Bosangani et nous emmenons toute une classe en bus dans la ville sans qu'il soit jamais question d'argent, comme s'il s'agissait spontanément ici d'un échange égal et d'autant plus agréable qu'il est gratuit. Dans tous les cas, évidemment, nous nous engageons à fournir aux divers acteurs une copie du film. Beaucoup plus rarement, nous offrons des copies de rushes (faciles à promettre, toujours difficiles à faire).

Dernière anecdote : le seul filmage impossible est celui du golf de Kinshasa, vestige intact de l'ère coloniale, coin de paradis perdu en plein cœur de la ville dévastée. L'entrée nous est refusée. Nous filmons de loin et de haut, en catimini, comme dans un reportage clandestin sous les pires dictatures. D'instinct, les golfeurs, blancs ou noirs, se méfient de leur image.

\section{Kinshasa 2002}

Sujet : Kinshasa. Film de commande pour la collection «L'écume des villes ». Pas de repérage cette fois. Celui-ci, en somme, a été fait en 2000. Nous savons où nous mettons les pieds, même si Kabila père a été assassiné entre-temps et si le colonel Kapend est en prison, accusé de cet assassinat... Mais nous n'avons pas le même besoin d'un aval institutionnel, notre sujet n'a rien de politique. Nous ferons donc avec les autorisations habituelles et relatives. Mais surtout nous retravaillons avec Paul Maseke, nous en faisons même un des personnages du film. Il raconte son quartier, Kingasani. Il introduit d'autres personnages. Contrairement au film de 1985, nous ne sommes jamais en scène, mais nous ne sommes pas pour autant dans l'illusion d'un réel non filmé. Chaque situation est artificielle, chacun des personnages, qui nous guide, qui nous fait découvrir un coin de la ville, une ambiance, un artiste, qui nous initie à la « débrouillation » kinoise, s'adresse directement à la caméra. On fait comme si on n'était pas là, mais la caméra occupe une place centrale, tantôt miroir comme en 1985, tantôt subjective, se glissant dans le regard du guide. Elle se fait apostropher, comme d'habitude.

Nous renouvelons l'expérience de tournage d'il y a deux ans, l'obscure protection en moins. Plus que jamais, il faut baliser, déminer le terrain. Le sujet ne permet guère de faux-fuyants : c'est bien la ville qu'on filme, et donc les gens. Kinshasa apparaît plus anarchique que jamais. Episodes innombrables d'interpellations policières sous les prétextes les plus divers, malgré les précautions, les visites préventives. Les affaires se règlent généralement avec des cadeaux, cartes téléphoniques ou boissons sucrées. Nous tombons dans un piège tendu par des fonctionnaires de l'Agence nationale de renseignement (ANR). Je paie cent USD pour une autorisation spéciale de tournage dans certains lieux décrétés stratégiques (sur le fleuve, à la gare et dans un train notamment), autorisation que je ne recevrai jamais. Le chantage dure trois jours : encore cent USD, et vous aurez le laissez-passer du siècle. Notre refus nous expose à des représailles, il faudra brouiller les pistes et mener très discrètement (!) certains tournages.

Il n'y a pas que les entraves policières. Toute personne disposant d'un képi, d'un cachet, d'un brassard ou simplement de culot et de bagou est susceptible de vous empêcher de travailler et de vous réclamer son dû. L'autorisation délivrée par la direction de la Société des chemins de fer, après de laborieuses démarches, ne garantit rien, ni la présence d'un haut fonctionnaire dûment habilité : quand nous débarquons à six heures du matin à Kasangulu 
pour prendre le train vers Kinshasa, tout commence par une explication orageuse entre les protagonistes congolais du tournage et un sous-chef de gare. Moyennant paiement discret du sous-chef par l'un d'entre eux (que nous rembourserons), le train partira avec une heure de retard. Dans tous les cas, il vaut mieux négocier directement soi-même. Je travaille toujours en tandem avec Maseke. Il faut croiser les arguments, cerner le point de rupture de l' « adversaire » et surtout s'assurer que l'interlocuteur est le bon, celui qui détient l'ultime pouvoir d'empêchement ${ }^{11}$.

Ce tournage révèle aussi une ville de Kinshasa plus violente qu'auparavant, l'excès et l'agressivité à fleur de peau, à l'égard des Blancs notamment. L'impopularité de la MONUC n'y est sans doute pas pour rien, mais il y a surtout cette misère sans espoir et ces humiliations sans fin. Le voyage en train de Kasangulu au Centre-ville, à travers l'interminable banlieue kinoise, est une extraordinaire révélation à cet égard. Le caméraman et le preneur de son sont coincés, enserrés à l'intérieur. La réalisatrice et moimême sommes à l'avant de la locomotive. Quand le train arrive dans les premières gares, encore rurales, nous sommes accueillis, salués en héros par la foule. Au fur et à mesure que nous approchons du centre, les cris se font de plus en plus hostiles. Et quand nous filmons le passage du même train deux jours plus tard, à l'approche de la gare centrale, c'est une explosion de violence. Le caméraman manque d'être écharpé par des dizaines de jambes tendues à l'extérieur des voitures, il est sauvé in extremis par Maseke.

Au cœur de la ville, où tout se concentre, quelque chose ne semble plus supportable dans le regard du Blanc.

\section{«Pourquoi vous filmez?"}

La question, posée en 1985, est toujours valable. La seule vraie constante dans ces diverses expériences est la relation des Congolais à la caméra professionnelle. Celle-ci est surtout perçue comme un objet étranger, blanc, extérieur, comme une «caméra de surveillance », un objet décidément post-colonial ${ }^{12}$. Dans les tableaux du peintre kinois Ekunde (entre autres), qui représentent des scènes de la vie urbaine, il y a toujours dans un coin un type en train de filmer la scène, toujours blanc, souvent roux. La caméra semble prolonger indéfiniment le regard colonial, l'œil du Blanc sur le Noir.

A cet égard, il n'y a pas de différence entre Mobutu et la plupart des Congolais. Je pense que quand ils sont filmés, ils se montrent d'abord, ou se dérobent, au regard européen, peu importe que ce film soit autant vu en Afrique qu'en Europe, voire même davantage. Et il y a tout un comportement lié à cet état de fait, qui tient de l'insoumission, de l'agressivité, de la séduction. La caméra est violente, c'est une arme technologique, il faut se l'approprier. La caméra lèse et dépouille, il faut l'envoûter, l'apprivoiser. La caméra est une machine à illusions, elle embellit, elle refait le monde : en cela, l'Afrique n'a pas fini de s'en emparer. Dans tous les films tournés à Kinshasa, il y a une vibration singulière, un art du verbe, un détournement de l'image par la parole. C'est en parlant que le Kinois crève l'écran, sans souci de paraître, contrairement à sa réputation, ou

11. Ainsi pour le tournage sur le fleuve, faute d'avoir négocié au bon niveau, nous avons perdu l'argent versé au pilote du bateau qui se faisait fort de passer tous les barrages administratifs.

12. Idem au Burundi, le pays d'Afrique où j'ai le plus filmé après le Congo-Zaïre. Il y a au Burundi, pays très différent de ce dernier à de nombreux égards, une méfiance extraordinaire, volontiers paranoïaque, à l'égard de la caméra, soupçonnée de tous les complots. 
peut-être conformément à sa réputation, tant il n'a guère d'inquiétude à cet égard. Les questions d'argent, qui prennent tant d'importance dans notre démarche, sont finalement secondaires pour ceux que nous filmons : c'est la caméra qui les intéresse, c'est inverser les rôles, casser l'image, parler au monde, se regarder fièrement.

\section{Notice filmographique}

1985 : «Kin 85 ». Edition spéciale Journal télévisé. Equipe : Didier Lannoy, Michel Rouserez, Raymond Wieme.

1990 : «Le léopard blessé ». Magazine « Plein Cadre ». Equipe : Michel Boulogne, Guy Lejeune.

1992: «Ailleurs au Congo - Les orphelins du Katanga ». Magazine «Plein Cadre ». Equipe : Jean-Michel Germys, Patrick Van Nyen, Guy Tournay.

1994 : « Maréchal, revoilà !». Magazine «L'Hebdo ». Equipe : Isabelle Christiaens, Bernard Dellis, Michel Rouserez, Alex Vander Perren.

1998 : « Missionnaire chez les Blancs ». Coproduction Arte - RTBF - Dérives pour la collection « Que reste-til des colonies? ». Equipe : Isabelle Christiaens, Vincent Fooij, Paul Heymans.

2000 : «Indépendance cha cha ». Coproduction RTBF - VRT. Equipe : Isabelle Christiaens, Vincent Fooij, Serge Schots.

2002 : «Kinshasa ». Coproduction RTBF - Les Films d'Ici - Saga Films pour la collection «L'écume des villes ». Equipe : Isabelle Christiaens, Michel Boulogne, Serge Schots. 